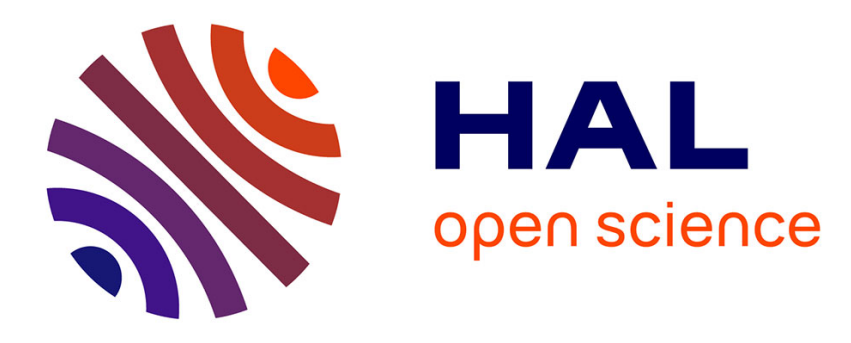

\title{
Extrapolation of DEM simulations to large time scale. Application to the mixing of powder in a conical screw mixer
}

Xavier Bednarek, Sylvain Martin, Abibadou Ndiaye, Véronique Peres, Olivier Bonnefoy

\section{To cite this version:}

Xavier Bednarek, Sylvain Martin, Abibadou Ndiaye, Véronique Peres, Olivier Bonnefoy. Extrapolation of DEM simulations to large time scale. Application to the mixing of powder in a conical screw mixer. Chemical Engineering Science, 2019, 197, pp.223-234. 10.1016/j.ces.2018.12.022 . hal-01968891

\section{HAL Id: hal-01968891 https://hal.science/hal-01968891}

Submitted on 26 Feb 2019

HAL is a multi-disciplinary open access archive for the deposit and dissemination of scientific research documents, whether they are published or not. The documents may come from teaching and research institutions in France or abroad, or from public or private research centers.
L'archive ouverte pluridisciplinaire HAL, est destinée au dépôt et à la diffusion de documents scientifiques de niveau recherche, publiés ou non, émanant des établissements d'enseignement et de recherche français ou étrangers, des laboratoires publics ou privés. 


\title{
Extrapolation of DEM simulations to large time scale. Application to the mixing of powder in a conical screw mixer
}

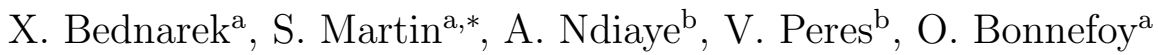 \\ ${ }^{a}$ Mines Saint-Etienne, Univ Lyon, CNRS, UMR 5307 LGF, Centre SPIN, F - 42023 \\ Saint-Etienne France \\ ${ }^{b}$ MELOX ORANO
}

\begin{abstract}
The paper proposes an original algorithm which allows a long time scale extrapolation of DEM results at a very low computational cost. This algorithm can be adapted to any periodic processes. In this study, it is applied to the mixing process of powders within a conical screw mixer. The results are then compared with long time DEM simulations. It appears that this method is able to predict the DEM results with a very good accuracy.

Keywords:

Mixing powders, DEM, Large time scale simulation, Extrapolation
\end{abstract}

\section{Introduction}

Over the last decades, the Discrete Element Method (DEM) Cundall and Strack, 1979) has become a major tool to simulate the behavior of granular media. Since it is the only method able to consider the discrete aspect of materials, it is well designed to represent the mixing of powders. Thus, sev-

*E mail address: sylvain.martin@emse.fr 
eral blender techniques have been investigated through DEM: rotating drums (Mishra et al., 2002; Yang et al., 2008; Liu et al., 2013), screw conveyor-mixers (Hou et al., 2014, Pezo et al., 2015), V-blenders (Lemieux et al., 2007, 2008; Kuo et al., 2002), Turbula ${ }^{\circledR}$ (Marigo et al., 2011), static mixers (Pezo et al., 2016) and Nauta blenders (Golshan et al., 2017).

However, one of the main limits of the method is its high computational cost (Bertrand et al. 2005). DEM is indeed able to represent a few millions particles (Radeke et al., 2010), which is far from the real number of grains involved in industrial mixing processes. Moreover, only a few minutes of real time can be reached for such simulations.

Hence, there is a crucial need to develop new methods able to extrapolate the DEM results to large space and time scales. Several studies have been dedicated to overcome this limit. A first idea is to use a coarse grain model Sakai and Koshizuka (2009), which consists in considering coarse grains, the properties of which reproduce the average behavior of small grains assemblies. Another approach consists in simulating the granular media with continuous models. Discrete and continuous approaches can also be coupled to combine advantages of both methods, using DEM in high shear rate regions and a continuous method elsewhere (Chen et al., 2016). However, this method is also limited to the simulation of convective mixing. There are also very promising works, based on a continuous approach coupled with a scalar-transport-equation, that simulates the concentration of the different particles species. The velocity field is interpolated from DEM results and the transport equation takes into account the different mixing mechanisms, i.e. advection and diffusion. Segregation velocity is also considered. The differ- 
ent adjustable parameters, like diffusion coefficient and segregation velocity, are fitted from DEM simulations. For more information on this approach, see the following references: Fan et al. (2014); Schlick et al. (2015, 2016). If this approach seems very promising, it has only been applied to very simple system so far.

In this context, the simulation of monodisperse powders remains very interesting. It does not exhibit the full complexity of industrial processes but allows the implementation of simple numerical approaches which can give very relevant results. For example, Doucet et al. (2008) proposed to simulate the mixing of powders with a DEM-based stationary Markov process, which permits to extrapolate the DEM results to large time scale.

This paper an original algorithm which allows a long time scale extrapolation of DEM results at a very low computational cost. Contrary to the works of Doucet et al. (2008), the present method is fully deterministic and meshless. This original algorithm can be adapted to any periodical process with monodisperse powder. It can also be implemented in coarse grain simulations to scale up the simulation time. In this paper, the method is applied to the simulation of a Conical Screw Mixer with one million particles.

\section{Material and method}

The method is very simple and can be generalized to many different systems. However, its implementation requires the following conditions:

- The targeted process is periodic,

- The particles are identical, 
- The granular medium is dense - i.e. the distance between the centers of neighboring particles is roughly equal to the diameter of a single particle.

Note that these two last conditions are soft constraints because they do not prevent the algorithm to be executed. Not fulfilling the second condition would lead to less accurate results. The method has indeed be designed assuming that all the particles have a similar behavior. Moreover, for different sized particles, segregation would not be taken into account. Then, not fulfilling the last condition would lead to minor errors. In the case of our screw mixer, the medium is very dense and this point is not an issue. However, the error could become significant for applications like spouted beds for which the bulk density is more heterogeneous.

The general idea of the method is first to run a short DEM simulation over a time-lapse which is greater than the period $T$ characteristic of the process. It should be noticed that $T$ is very different from the time step of the DEM simulation. $T$ is indeed only a property of the process, it is independent of the simulation parameters. It is generally several order of magnitude greater than the DEM resolution time step. For a mixing process with a rotating impeller, this time-lapse $T$ should be the rotation period of the screw impeller, while for a rotating drum this could be the interval between two avalanches. We note $\alpha \geq 1$ the number of period $T$ which is simulated:

$$
t_{1}-t_{0}=\alpha T
$$


where $t_{0}$ is the initial time fixed by the user and $t_{1}$ is the final time of the DEM simulation. Then, the purpose of the extrapolation is simply to repeat the track followed by particles between $t_{0}$ and $t_{1}$ for predicting $t_{2}$ and then $t_{3}, t_{4} \ldots$

The repetition of the trajectories must be done on a single structure: that of the state $t_{1}$. One calls "structure" the set of the positions of particles for a given time. Since the structure at $t_{0}$ is not the same as that at $t_{1}$, it is necessary to bring the first one closer to the second. Thus, the crucial step of the algorithm consists in pairing the particles: each particle $j$ among the $N$ particles of the simulation at the starting time $t_{0}$, at position $\vec{r}_{j}\left(t_{0}\right)$, is associated with a particle $i$ of the ending time $t_{1}$, at position $\vec{r}_{i}\left(t_{1}\right)$, by finding the twinning function $f$, defined by Equation 2, which is bijective.

$$
\begin{aligned}
& f: \mid \llbracket 1, N \rrbracket \longrightarrow \llbracket 1, N \rrbracket \\
& i \longmapsto j=f(i)
\end{aligned}
$$

This twinning function has to be defined in such a way that $\vec{r}_{f(i)}\left(t_{0}\right)$ is sufficiently close to $\vec{r}_{i}\left(t_{1}\right)$. The meaning of "sufficiently" is discussed later in the text. A graphical representation of the pairing - $i e$ twinning - step is given in Figure 1. Note that the goal is to match particles at two distinct times. The fact that the trajectories are repeated implies the hypothesis that the paired particles must have the same behavior and therefore be mechanically identical. So in the ideal case they differ at most only in their color.

Finally, the position of the particle $i$ at time $t_{2}=t_{1}+\alpha T$ can be easily extrapolated: $\vec{r}_{i}\left(t_{2}\right)=\vec{r}_{f(i)}\left(t_{1}\right)$. In other words, particle $i$ will move, at time $t_{2}$, to the position of the particle $f(i)$ at time $t_{1}$. Let $f^{n}=f \circ \ldots \circ f$ be the composition of $n$ functions $f$, and $n$ a strictly positive integer. The 
position of particle $i$ at the time $t_{n+1}=t_{1}+n \alpha T$ is given by Equation 3 .

$$
\vec{r}_{i}\left(t_{n+1}\right)=\vec{r}_{f^{n}(i)}\left(t_{1}\right)
$$

Thus, knowing $f$, the extrapolation step can be reproduced many times at a very low computational cost. The general position vector and the displacement associated with the position during the time interval $\alpha T$ are indeed fixed over time.

\subsection{Example}

In order to make the method clearer, an example of the extrapolation approach is presented bellow. Lets consider a nondescript 9 particles system.

1. First of all, a DEM simulation is run. The initial steady state time $t_{0}$ is fixed and represented in the left picture of Figure 1, Then, the simulation is run up to $t_{1}=t_{0}+\alpha T$. The position of particle at time $t_{1}$ is shown in the middle picture of Figure 1.

2. Then, the twining function has to be determined. Looking at the positions of particles at time $t_{0}$ and $t_{1}$, it can be observed that particle 3 at time $t_{1}$ is closed to the position that was occupied by particle 1 at $t_{0}$. Thus $f(3)=1$. In the same way one has $f(1)=9, f(2)=7, f(4)=8$, $f(5)=6, f(6)=2, f(7)=3, f(8)=5$ and $f(9)=4$.

3. Finally, the positions of every particle can be extrapolated for the following times. Applying Equation 3, the position of particle 1 at time $t_{2}$ is predicted: $\vec{r}_{1}\left(t_{2}\right)=\vec{r}_{f(1)}\left(t_{1}\right)$ that is $\vec{r}_{9}\left(t_{1}\right)$. At time $t_{3}$, as one has $f(f(1))=4$, the position of particle 1 is predicted at $\vec{r}_{4}\left(t_{1}\right)$. Finally, since $f(f(f(1)))=8$, the position of particle 1 at time $t_{4}$ is predicted 
at $\vec{r}_{8}\left(t_{1}\right)$. Figure 2 shows these three extrapolated steps, particle 1 being in green, and follows the paths presented in Figure 1, moving the green color on the structure of state $t_{1}$. The same procedure is applied to all other particles.

The most time consuming part of the method is the pairing algorithm. It is described in the following section.

\subsection{Pairing algorithm}

The principle of the pairing algorithm is to couple particles of time $t_{0}$ with particles of time $t_{1}$ in order to get a set of the closest pairs. The aim is then to find $f$ that minimizes the objective function $F_{\text {obj }}$ defined as follows:

$$
F_{\text {obj }}=\sum_{i=1}^{N}\left\|\vec{r}_{i}\left(t_{1}\right)-\vec{r}_{f(i)}\left(t_{0}\right)\right\|
$$

The optimum pairing solution $f$ corresponds to the minimum of the sum of

the $N$ center to center distances $d_{\text {pair }_{i}}=\left\|\vec{r}_{i}\left(t_{1}\right)-\vec{r}_{f(i)}\left(t_{0}\right)\right\|$ between pairs of particles, $N$ being the number of particles in the simulation.

Since each particle has to be associated with another single particle, the full direct resolution of this problem would require the evaluation of the $N$ ! possible combinations. Hopefully, such a problem can be viewed as an Euclidean assignment problem (Rendl, 1988) and can be solved numerically by means of the Hungarian method introduced by Kuhn (1955). The input of this algorithm is the table of distances between each pair of particles candidate to coupling, whose size is $N^{2}$. This leads to a prohibitive computational memory and time calculation for large scale simulations with $N>10^{5}$ particles. Therefore, the number of combinations have to be substantially decreased. This aim is reached by introducing two simplifications: 
1. The method can be improved by avoiding taking into account pairs of particles, the distance between which is too high. Thus, the first simplification step consists in allowing a maximum number $N_{c}$ of pairing possibilities for each particle, excluding the furthest ones. This can be seen as a cutoff distance, similar to those implemented in the SPH or Molecular Dynamics methods, except that, in the present case, the cutoff criterion is firstly based on a maximum number of neighoring particles. Each particle $i$ at step $t_{1}$ has to get $N_{c}$ candidates $j$ at step $t_{0}$ and vice versa. Hence, the number of candidates is at least equal to $N_{c}$. Further explanations are given in Figure 3.

2. Simulation domain is divided into $N_{x} \times N_{y} \times N_{z}$ subdomains where $N_{x}, N_{y}$, and $N_{z}$ are respectively the number of slices in the $x, y$, and $z$ directions. The Hungarian algorithm is then run separately on every subdomain. This is kind of a domain decomposition which simplifies the resolution and also allows its parallel implementation.

In this study, with $N=10^{6}$ particles, the pairing algorithm has been run with $N_{c}=300$ and $N_{x}=N_{y}=N_{z}=10$.

It should be noticed that both the limitation to $N_{c}$ pairing candidates for each particle and the division of the global domain might not allow the convergence to the minimum for the objective function. Thus, the quality of the pairing solution should be evaluated before any extrapolation of the DEM results.

\subsection{DEM model}

A classical DEM model is used in this study. The particles are spherical and the particle size distribution (PSD) is uniform with a span $\left(d_{p}^{\max }-d_{p}^{\min }\right)$ 
equal to $5 \%$ of the mean diameter $d_{p}$. Since the purpose is to give an example of the extrapolation procedure, the contact law used here - the non-linear Hertz-Mindlin model - is not detailed but can be found elsewhere (Zhu et al., 2007). The simulation has been run with LIGGGHTS 3.1.0 (Kloss et al., 2012). The parameters used in the DEM simulation are given in Table 1.

Table 1: DEM parameters

\begin{tabular}{lccc}
\hline Parameter & Type & Value & Unit \\
\hline Number $N$ & Particles & $10^{6}$ & - \\
Mean radius $R=d_{p} / 2$ & Particles & 0.83 & $\mathrm{~mm}$ \\
Width of the PSD & Particles & 5 & $\%$ \\
Friction coefficient & Particle-Particle & 0.5 & - \\
& Particle-Wall & 0.2 & - \\
Rolling friction coefficient & Particle-Particle & 0.15 & - \\
& Particle-Wall & 0.01 & - \\
Restitution coefficient & Particle-Particle & 0.3 & - \\
& Particle-Wall & 0.9375 & - \\
Poisson ratio & Particle & 0.25 & - \\
Young modulus & Wall & 0.25 & - \\
& Particle & 0.1 & $\mathrm{GPa}$ \\
Density & Wall & 1 & $\mathrm{GPa}$ \\
& Particle & $10^{4}$ & $\mathrm{~kg} . \mathrm{m}^{-3}$
\end{tabular}

\subsection{Description of the conical screw mixer}

This method has been applied to the DEM simulation of powder mixing within a conical screw mixer. The later is a conic blender with a screw 
impeller. An accurate description of the mixer is given in Figure 4. The screw impeller rotates around the axis of the cone $\overrightarrow{e_{y}}$, going along the wall with an angular velocity $\omega_{a}$. This rotation will be labeled as primary rotation. The screw impeller also rotates around its own axis with a velocity $\omega_{s}$. This will be labeled as secondary rotation. In this study, $\omega_{s}=30 \omega_{a}$.

\subsubsection{Filling procedure}

The conical vessel is filled with particles using a classical DEM procedure: initial positions of particles are randomly chosen in the upper part of the cone and particles are then allowed to fall by gravity. The filling step is considered to be accomplished when the mean translational velocity is bellow $10^{-4} \mathrm{~m} . \mathrm{s}^{-1}$. Friction coefficients and rolling friction coefficients are set to zero to ensure that the upper free surface is almost flat at the beginning of the mixing process.

\subsection{Evaluation of the mixing quality}

\subsubsection{Mixing indices}

Multiple indices are available in the literature to evaluate the mixing quality for at least two components: the component "of interest" and the other one that can regroup several component excluding of course the one "of interest". The Lacey index (Lacey, 1954) $I_{L}$ is the most common. It is defined as follows:

$$
I_{L}=\frac{\sigma_{0}^{2}-\sigma_{m}^{2}}{\sigma_{0}^{2}-\sigma_{r}^{2}}
$$

where $\sigma_{m}^{2}$ is the variance of the composition of the component of interest over

the sampling, $\sigma_{0}^{2}$ is the variance of the fully segregated state: $\sigma_{0}^{2}=p(1-p)$, with $p$ the ratio of particles of the component of interest in the whole mixture, 
$\sigma_{r}^{2}$ the variance of the random system: $\sigma_{r}^{2}=\frac{p(1-p)}{n_{s}}$ with $n_{s}$ the number of particles - regardless the nature of the particle - in each sample.

This index tends rapidly to 1 with respect to the mixing quality. Hence, we chose to also calculate the Ashton index $I_{A}$ (Ashton and Valentin, 1966) which allows a better discrimination between mixing states when the system is almost homogeneous:

$$
I_{A}=\frac{\log \left(\frac{\sigma_{0}^{2}}{\sigma_{m}^{2}}\right)}{\log \left(\frac{\sigma_{0}^{2}}{\sigma_{r}^{2}}\right)}
$$

This index also tends to 1 but slower to the Lacey index. It should be noticed that both indices depend strongly on the sampling protocol. Therefore, in this paper, the sampling is based on a 3D Voronoï diagram: 1000 particles are chosen randomly and 1000 samples are built around this particles. Each of the particles of the mixture is attached to the sample corresponding to the particle - among the 1000 initially selected - which is closest to it. This procedure is repeated 10 times and the indices presented are the average of the 10 mixing values thus obtained.

\subsubsection{Dispersion}

The dispersion is a mixing criterion that takes into account both convective and diffusive mixing mechanisms. In order to quantify it, $N$ clusters are considered. Each cluster $i$ is composed of a central particle $i$ and neighbouring particles $j$. The neighbourhood limited to particles $j$ that are separated from the central particle by a surface-to-surface distance of less than a mean radius i.e. $R=8.310^{-1} \mathrm{~mm}$. This translates into:

$$
\left\|\vec{r}_{j}-\vec{r}_{i}\right\|<R_{i}+R_{j}+R
$$


Each particle is often part of several clusters. The distance of dispersion $d_{\text {disp }}^{i}$ of one single cluster $i$ is defined as the average distance between the central particle $i$ and its $M_{i}$ neighbouring particles $j$ at a time $t$ minus the same mean distance but $\Delta t_{\text {disp }}$ earlier.

$$
d_{\text {disp }}^{i}\left(t ; \Delta t_{\text {disp }}\right)=\sum_{j=1}^{M_{i}}\left\|\vec{r}_{j}(t)-\vec{r}_{i}(t)\right\|-\left\|\vec{r}_{j}\left(t-\Delta t_{\text {disp }}\right)-\vec{r}_{i}\left(t-\Delta t_{\text {disp }}\right)\right\|
$$

Most of the time, such a distance is positive but it can also be negative if any compression of the cluster occurs. Golshan et al. (2017) used a similar criteria to define their tracers. Here, every particle is considered as the center of a cluster.

Finally, the dispersion $d_{\text {disp }}$ is interpolated to a tetrahedral mesh. This allows us to build a continuous dispersion field, for a given duration $\Delta t_{\text {disp }}$, over the volume of the conical screw mixer.

\section{Results and discussion}

The extrapolation method decribed above has been applied to the mixing process of powder.

The standard DEM simulation has been run for a full primary revolution of the screw impeller in the conical mixer, which corresponds to $30 \mathrm{~s}$ of physical time and 30 rotations of the screw impeller around its own axis (with $\left.\omega_{s}=60 \mathrm{rpm}\right)$.

During post-processing, four initial configurations were created by assigning different colors to the particles regarding their initial positions. The different configurations are labeled according to the number of slices in the 
corresponding direction, respectively $2 x, 2 y, 2 z$ and $8 y$. For example, $8 y$ means that initialy there are eight layers of particles stacked on top of each other in the $y$ direction. These configurations are represented in Figure 5. Since only one type of particle is mixed, mixing indices cannot be calculated without any coloration procedure.

Finally, the extrapolation algorithm has been run to predict the two next primary revolutions of the screw impeller.

\subsection{Determining the steady state}

Extrapolation requires that the system be in a permanent state. It means that the velocity field has to reach its periodic state with respect to the screw rotation. After the filling step, the simulation starts with an acceleration step, which is due to the inertia of the system. Moreover, the shape of the upper free surface of the granular packing can evolve slightly between the static and dynamic state. This explains why applying the pairing algorithm before reaching the steady state would increase significantly the error of the pairing solution, particularly near the top of the mixer.

Therefore, the beginning steady state can be determined by considering the average velocity of the particles and the torque acting on the screw impeller, along its axis of rotation. It is shown in Figure 6 that the steady state is reached after ten secondary revolution of the impeller i.e. $10 \mathrm{~s}$ of mixing. In this study, the initial state was set at $t_{0}=21 \mathrm{~s}$ for the extrapolation algorithm.

One natural time period of the process is $T=1 \mathrm{~s}$, the period of rotation of the screw. We take $\alpha=9$ in Equation 1 and the final state of the simulation is at $t_{1}=30 \mathrm{~s}$. The extrapolation is so conducted with a $\alpha T=9 \mathrm{~s}$ step which 
corresponds to 9 full revolutions of the screw impeller.

\subsection{Accuracy of the pairing algorithm}

If the pairing algorithm is executed directly on steps $t_{0}$ and $t_{1}$, this will lead to poor results because of the non alignment of the empty volumes created by the screw impellers that are not in the same position for the two time steps. In that case, it is a critical point to superimpose the screws before the execution of the algorithm. Thus the whole state at time $t_{0}$ is rotated of an angle of $\alpha T \omega_{a}=-108^{\circ}$ around $\overrightarrow{e_{y}}$ - i.e. all particles and the meshes. The extrapolated time step $t_{n+1}$ is then obtained from extrapolated data after rotation by an angle $-n \times 108^{\circ}$ around $\overrightarrow{e_{y}}$.

The efficiency of the pairing algorithm can be evaluated by looking at the distances between paired particles. The most accurate results correspond to the smallest distances. The pairing distance distribution is given in Figure 7 and Table 3. It appears that the distance $d_{\text {pair }}$ between paired particles is less than the mean diameter $d_{p}$ (resp. $1.2 d_{p}$ ) for 90\% (resp. 95\%) of the particles. This result is considered as rather good. However, it should be noticed that for a very few particles, the pairing distance increases up to 27.7 times the mean diameter $d_{p}$. This can be explained by the slight evolution of the free surface shape between $t_{0}$ and $t_{1}$ and the domain decomposition into $N_{x} \times N_{y} \times N_{z}=1000$ domains in order to accelerate the execution of the pairing algorithm. This can be seen in Figure 8, It is also interesting to notice that this algorithm works well in our dense packing simulation, but would lead to a higher error for looser packings. The pairing algorithm was run on a single computer with a $4 \times 3.5 \mathrm{GHz}$ intel i5 processor and took about $10 \mathrm{~h}$ for $10^{6}$ particles. 


\subsection{Extrapolation of the DEM results}

The extrapolation algorithm has been run to predict the two following primary revolutions of the screw impeller. To validate the extrapolation results, a DEM simulation has also been run for two extra full primary revolutions.

The time gains over the full simulations are summed up in Table 2. The later presents typical CPU times for full DEM simulation compared with the extrapolation protocole, and gives the ratio of CPU time between DEM simulation and extrapolation with respect to the number of impeller primary convolution. Hence, the last column represents the gain factor which is expected. Since the extrapolation process requires a short initial DEM simulation and then the resolution of the pairing algorithm, this method becomes very interesting for long time extrapolation. Here, it has been limited to two extra revolutions because it was not possible to run longer DEM simulation for comparison. Thus, once the pairing algorithm has been solved, the extrapolation step for a full primary revolution takes about 1 min while it takes $\approx 5 . \times 10^{3} h r$ of CPU time for DEM. This emphasizes the major gain reached by the extrapolation approach.

However, attention should be drawn to the fact that those results give only orders of magnitude. They could evolve slightly according to the computer configuration and optimization of the codes. In our study, DEM simulation were run with a $2.6 \mathrm{GHz}$ cluster with 40 parallel threads while both pairing and extrapolation algorithms were run on a single $4 \times 3.5 \mathrm{GHz}$ intel is CPU.

For comparison, the cross sectional views of DEM and extrapolated results are given in Figure 9 and Figure 10 for the different configurations. All the results for extrapolation and DEM look very similar. For example, 
Table 2: Comparison between typical CPU times (hr) between DEM simulation and extrapolation method with respect to the number of impeller primary convolution. On the last line, +1 represent an extra primary convolution once the pairing algorithm has been resolved. For example, the CPU time for 3 full primary convolutions, is equal to the CPU time for 2 full convolutions plus the time for 1 extra primary convolution.

\begin{tabular}{ccccccc}
\hline \multirow{2}{*}{$\begin{array}{c}\text { Primary } \\
\text { convolution }\end{array}$} & DEM (hr) & \multicolumn{5}{c}{ Extrapolation method (hr) } \\
\hline 1 & $5 \times 10^{3}$ & $5 \times 10^{3}$ & 0 & 0 & $5 \times 10^{3}$ & 1 \\
\hline 2 & $1 \times 10^{4}$ & $5 \times 10^{3}$ & $4 \times 10^{1}$ & $1.5 \times 10^{-2}$ & $5.04 \times 10^{3}$ & 2 \\
\hline+1 & $5 \times 10^{3}$ & & $1.5 \times 10^{-2}$ & & $3 \times 10^{5}$ \\
\hline
\end{tabular}

the shape of the red layer of the $8 y$ configuration is well reproduced at the different stages of the simulation.

In order to get a more accurate comparison between extrapolation and DEM simulations results, Lacey and Ashton indices have been calculated for the four different initial configurations.

The results are presented repectively in Figure 11 and Figure 12 for Lacey indices and in Figure 13 and Figure 14 for Ashton indices. Those make it clear that the mixing kinetic is correctly predicted by the extrapolation scheme. To go further and evaluate the accuracy of the method, the relative difference, between extrapolated and DEM results for the Lacey and Ashton indices is given in Figure 15 and Figure 16 respectively. It appears that the error remains lower than $0.7 \%$ for the Lacey index and lower than $1.5 \%$ for the Ashton index, for all the configurations. This shows that the extrapolation algorithm reproduces very accurately the different mixing mechanisms.

Finally, the dispersion field was calculated for $\Delta t_{d i s p}=\alpha T=9 \mathrm{~s}$, start- 
ing from $t_{1}$, for DEM results and extrapolation results. This parameter is very interesting for two reasons: (1) the pairing algorithm adds an artificial dispersion by pairing the particles with particles that are not exactly at the same location and (2) it describe in details the diffusion mechanism. Thus, the comparison between the dispersion fields for DEM and extrapolated results is given in Figure 17. The relative error is presented in Figure 18 and Table 4. We can see that the relative differences can be high, greater than 1000\%. Two reasons explain this observation. First, when DEM dispersion is near zero, the dispersion added by the extrapolation algorithm is comparatively high even it is if low in absolute value. Secondly, the highest pairing distances near the free surface lead to a poorer rendering of the dispersion magnitude as shown in Figure 17.

In order to investigate the influence of $\alpha$, another extrapolation has been made with $\alpha=1$ - ie with an extrapolation step of $1 \mathrm{~s}$. In that case, the relative errors are larger, about $5 \%$ percent for the Lacey index and $8 \%$ percent for the Ashton index. This is simply explained by the fact that the prediction of the same time $t$, needs 9 times more steps with $\alpha=1$ than with $\alpha=9$. This leads to greater errors comming from the dispersion error accumulated at each extrapolation step. Thus a larger $\alpha$ decreases the cumulative error but leads to less points to describe the evolution of the extrapolated mixture.

\section{Conclusion}

An original method was presented for the long time extrapolation of DEM simulation by using a pairing algorithm. The results show an extremely 
good agreement between extrapolated and DEM results. Thus, this method is able to predict accurately long time results at a very low computational cost compare to a full DEM simulation. This method has been applied to the simulation of mixing in a conical screw mixer but it could easily be applied to any other periodic mixers like rotating drums, four bladed mixers, Turbula $\mathbb{R}$ , V-blenders... The method could also be extended to simulate more complex processes, like spouted beds, the particles of which have a cyclic behavior.

\section{References}

Ashton, M., Valentin, F., 1966. Mixing of powders and particles in industrial mixers. Transactions of the institution of chemical engineers and the chemical engineer 44, T166.

Bertrand, F., Leclaire, L.A., Levecque, G., 2005. DEM-based models for the mixing of granular materials. Chemical Engineering Science 60, 2517-2531. URL: http://www.sciencedirect.com/science/article/ pii/S0009250904009492, doi:https://doi.org/10.1016/j.ces.2004. 11.048 .

Chen, X., Wang, J., Li, J., 2016. Multiscale modeling of rapid granular flow with a hybrid discrete-continuum method. Powder Technology 304, 177-185. URL: http://www.sciencedirect.com/ science/article/pii/S0032591016304983, doi:https://doi.org/10. $1016 / j$.powtec. 2016.08 .017 .

Cundall, P.A., Strack, O.D., 1979. A discrete numerical model for granular assemblies. Geotechnique 29, 47-65. 
Doucet, J., Hudon, N., Bertrand, F., Chaouki, J., 2008. Modeling of the mixing of monodisperse particles using a stationary DEM-based Markov process. Computers \& Chemical Engineering 32, 1334-1341.

Fan, Y., Schlick, C.P., Umbanhowar, P.B., Ottino, J.M., Lueptow, R.M., 2014. Modelling size segregation of granular materials: the roles of segregation, advection and diffusion. Journal of Fluid Mechanics 741, 252-279.

Golshan, S., Zarghami, R., Norouzi, H.R., Mostoufi, N., 2017. Granular mixing in nauta blenders. Powder Technology 305, 279-288.

Hou, Q., Dong, K., Yu, A., 2014. DEM study of the flow of cohesive particles in a screw feeder. Powder Technology 256, 529-539.

Kloss, C., Goniva, C., Hager, A., Amberger, S., Pirker, S., 2012. Models, algorithms and validation for opensource DEM and CFD-DEM. Progress in Computational Fluid Dynamics, an International Journal 12, 140-152.

Kuhn, H.W., 1955. The Hungarian method for the assignment problem. Naval Research Logistics (NRL) 2, 83-97.

Kuo, H., Knight, P., Parker, D., Tsuji, Y., Adams, M., Seville, J., 2002. The influence of DEM simulation parameters on the particle behaviour in a V-mixer. Chemical Engineering Science 57, 3621-3638.

Lacey, P.M.C., 1954. Developments in the theory of particle mixing. Journal of applied chemistry 4, 257-268.

Lemieux, M., Bertrand, F., Chaouki, J., Gosselin, P., 2007. Comparative 
study of the mixing of free-flowing particles in a V-blender and a binblender. Chemical Engineering Science 62, 1783-1802.

Lemieux, M., Léonard, G., Doucet, J., Leclaire, L.A., Viens, F., Chaouki, J., Bertrand, F., 2008. Large-scale numerical investigation of solids mixing in a V-blender using the discrete element method. Powder Technology 181, $205-216$.

Liu, P., Yang, R., Yu, A., 2013. DEM study of the transverse mixing of wet particles in rotating drums. Chemical Engineering Science 86, 99-107.

Marigo, M., Cairns, D., Davies, M., Ingram, A., Stitt, E., 2011. Developing mechanistic understanding of granular behaviour in complex moving geometry using the Discrete Element Method: Part B: Investigation of flow and mixing in the Turbulaß mixer. Powder technology 212, 17-24.

Mishra, B., Thornton, C., Bhimji, D., 2002. A preliminary numerical investigation of agglomeration in a rotary drum. Minerals Engineering 15, $27-33$.

Pezo, L., Jovanović, A., Pezo, M., Čolović, R., Lončar, B., 2015. Modified screw conveyor-mixers - Discrete element modeling approach. Advanced Powder Technology 26, 1391-1399.

Pezo, M., Pezo, L., Jovanović, A., Lončar, B., Čolović, R., 2016. DEM/CFD approach for modeling granular flow in the revolving static mixer. Chemical Engineering Research and Design 109, 317 - 326. URL: http://www.sciencedirect.com/science/article/pii/ 
S0263876216000666, doi:https://doi.org/10.1016/j.cherd.2016.02. 003.

Radeke, C.A., Glasser, B.J., Khinast, J.G., 2010. Large-scale powder mixer simulations using massively parallel architectures. Chemical Engineering Science 65, 6435 - 6442. URL: http://www.sciencedirect. com/science/article/pii/S0009250910005737, doi:https://doi.org/ $10.1016 /$ j.ces.2010.09.035.

Rendl, F., 1988. On the Euclidean assignment problem. Journal of Computational and Applied Mathematics 23, 257 - 265. URL: http: //www.sciencedirect.com/science/article/pii/0377042788900015, doi:https : //doi.org/10.1016/0377-0427(88)90001-5.

Sakai, M., Koshizuka, S., 2009. Large-scale discrete element modeling in pneumatic conveying. Chemical Engineering Science 64, 533-539.

Schlick, C.P., Fan, Y., Isner, A.B., Umbanhowar, P.B., Ottino, J.M., Lueptow, R.M., 2015. Modeling segregation of bidisperse granular materials using physical control parameters in the quasi-2D bounded heap. AIChE Journal 61, 1524-1534.

Schlick, C.P., Isner, A.B., Freireich, B.J., Fan, Y., Umbanhowar, P.B., Ottino, J.M., Lueptow, R.M., 2016. A continuum approach for predicting segregation in flowing polydisperse granular materials. Journal of Fluid Mechanics 797, 95-109. doi:10.1017/jfm.2016.260.

Yang, R., Yu, A., McElroy, L., Bao, J., 2008. Numerical simulation of particle 
dynamics in different flow regimes in a rotating drum. Powder Technology $188,170-177$.

Zhu, H., Zhou, Z., Yang, R., Yu, A., 2007. Discrete particle simulation of particulate systems: Theoretical developments. Chemical Engineering Science 62, 3378-3396. URL: http://www.sciencedirect. com/science/article/pii/S000925090700262X, doi:https://doi.org/ $10.1016 /$ j.ces.2006.12.089. 

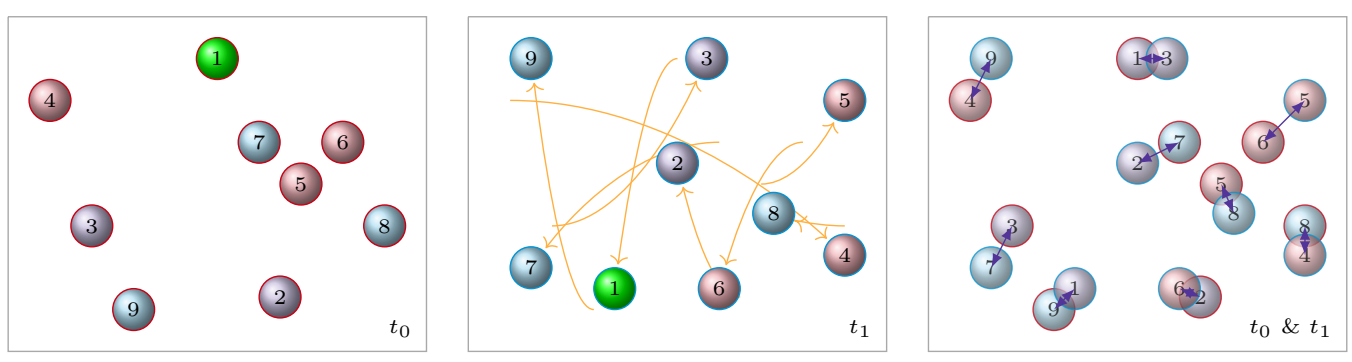

Figure 1: Representation of the pairing algorithm: particles position at time $t_{0}$ (left), $t_{1}$ (middle) and pairing step (right) where pairing distances are represented by doubleheaded arrows. Arrows (middle) arrows show the trajectories that have to be repeated by the extrapolation algorithm.
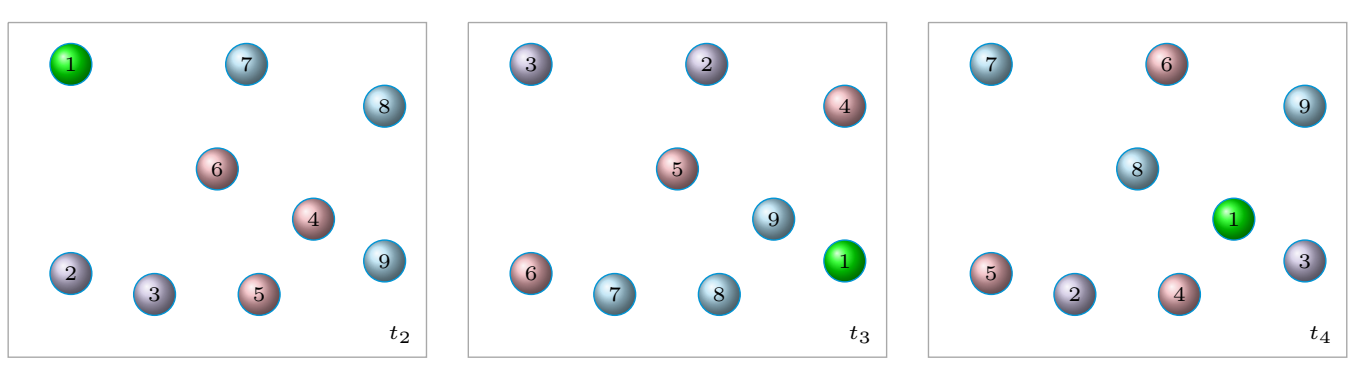

Figure 2: Representation of extrapolation algorithm, with the particle position at time $t_{2}$ (left), $t_{3}$ (middle) ans $t_{4}$ (right). Structure of state $t_{1}$ is conserved.

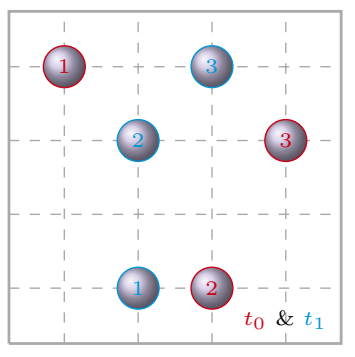

\begin{tabular}{|c|c|c|c|}
\hline & 1 & 2 & 3 \\
\hline 1 & & $\checkmark$ & $\checkmark$ \\
\hline 2 & $\checkmark$ & $\checkmark$ & \\
\hline 3 & & $\checkmark$ & $\checkmark$ \\
\hline
\end{tabular}

\begin{tabular}{|c|c|c|c|}
\hline & 1 & 2 & 3 \\
\hline 1 & & $\checkmark$ & $\checkmark$ \\
\hline 2 & $\checkmark$ & $\checkmark$ & \\
\hline 3 & $\checkmark$ & $\checkmark$ & $\checkmark$ \\
\hline
\end{tabular}

Figure 3: Example of pairing step (left) with $N c=2$ : searching $N_{c}$ candidates for particles of $t_{0}$ lead to the first table (middle) but particle $i=1$ has only one candidate; adding one more candidate to $i=1$ lead to the second table (right) with all particles having at least $N_{c}$ particles. 


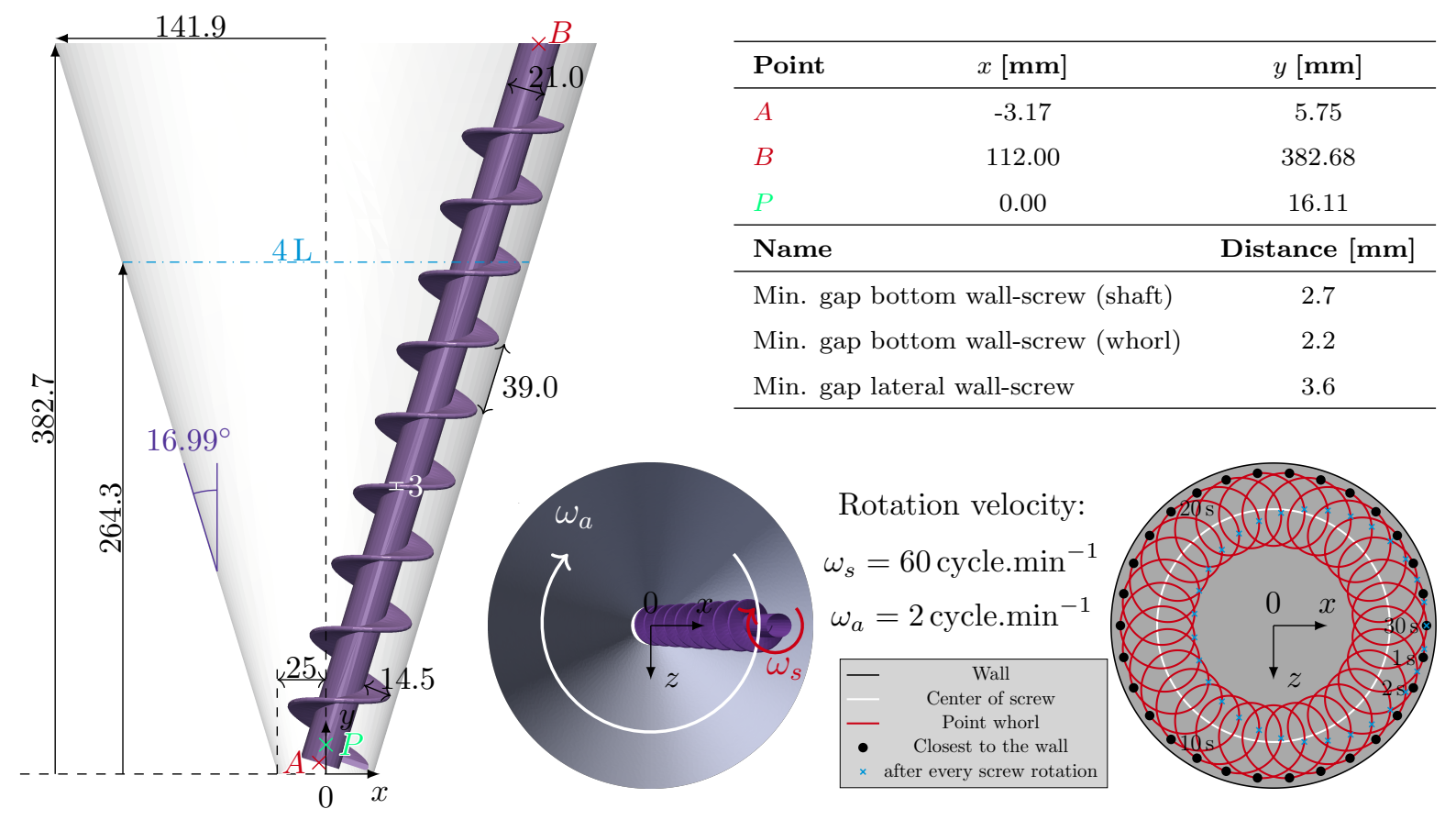

Figure 4: Characteristics of the conical screw mixer. Measures given in mm.
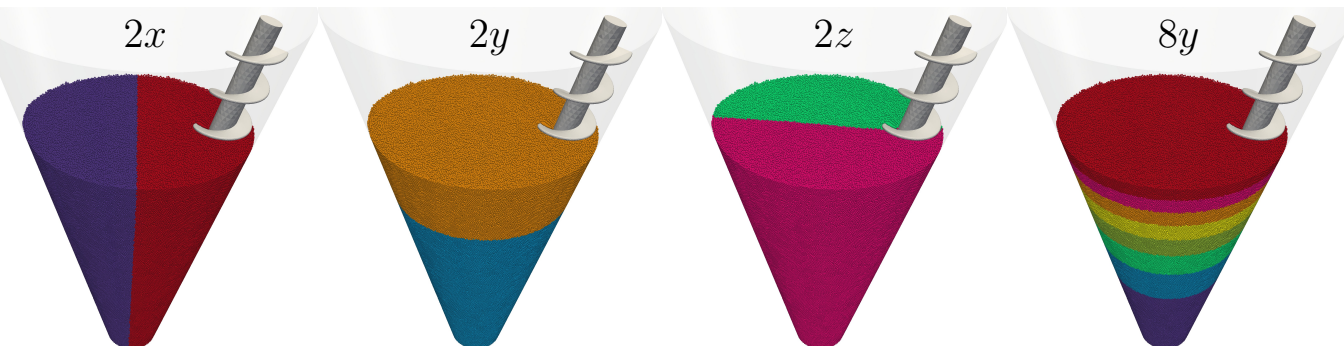

Figure 5: Representation of the four initial configurations 

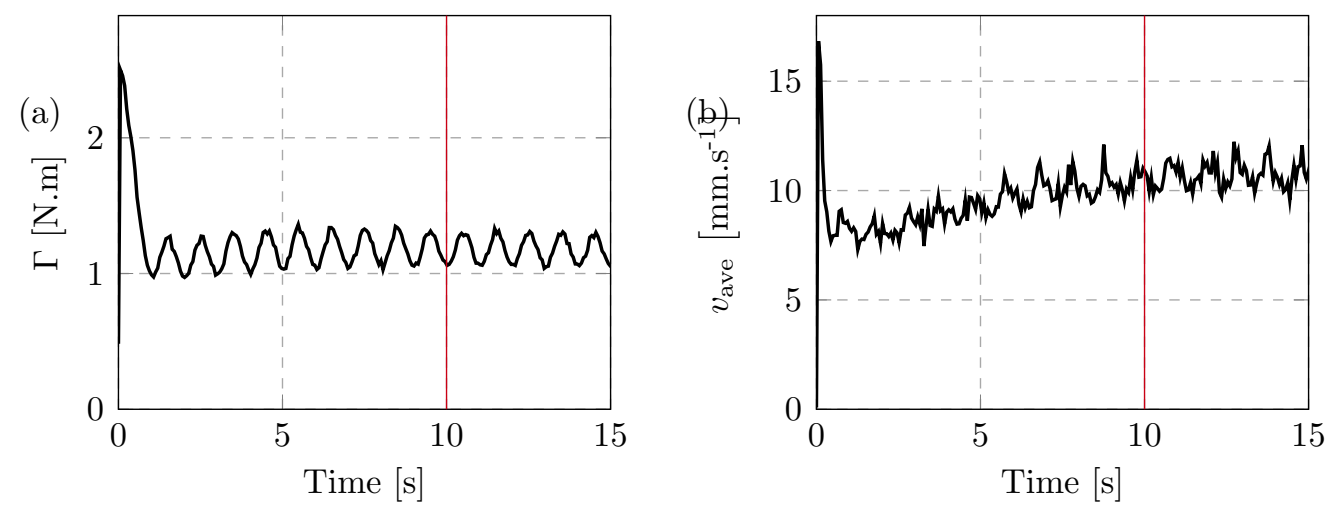

Figure 6: Determination of the steady state starting time regarding the torque $\Gamma$ acting on the screw impeller (left) and the average velocity $v_{\text {ave }}$ of the particles (right).

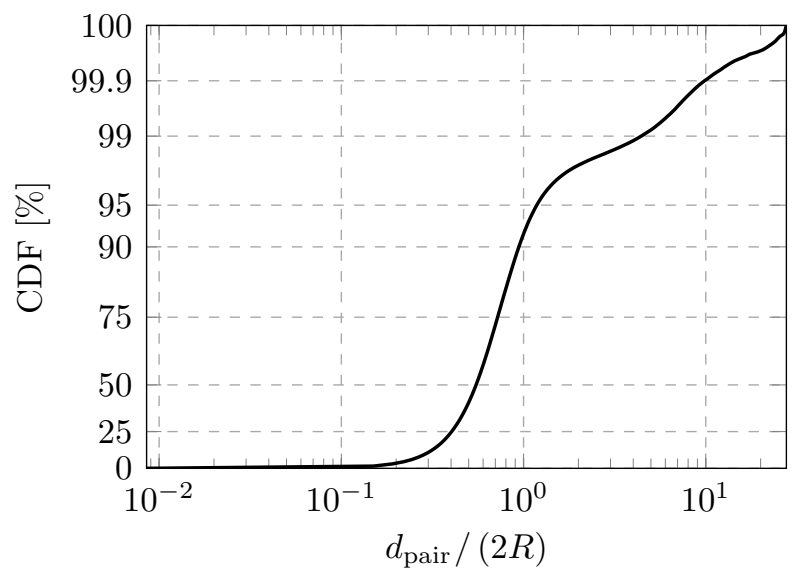

Figure 7: Representation of the pairing distances with cumulative distribution function (CDF) for the $N$ pair with an y-axis slightly dilated in order to get better indication on the highest distances. 
Table 3: Pairing distances

\begin{tabular}{cc}
\hline Frequency $[\%]$ & Relative distance $d_{\text {pair }} /(2 R)$ \\
\hline 10 & 0.29 \\
50 & 0.55 \\
90 & 0.94 \\
95 & 1.18 \\
99 & 4.42 \\
99.9 & 9.85 \\
99.99 & 18.52 \\
100 & 27.70 \\
\hline
\end{tabular}
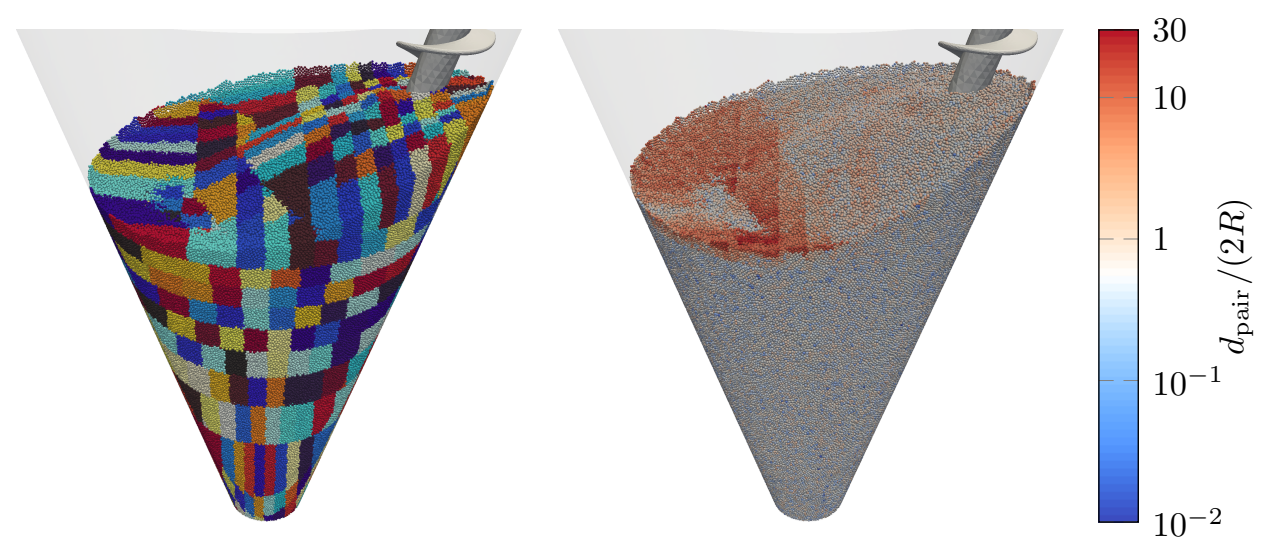

Figure 8: Pairing algorithm efficiency, considering pre-classification into $10 \times 10 \times 10$ domains (left), and regarding $d_{\text {pair }}$ repartition in $3 \mathrm{D}$ (right). 


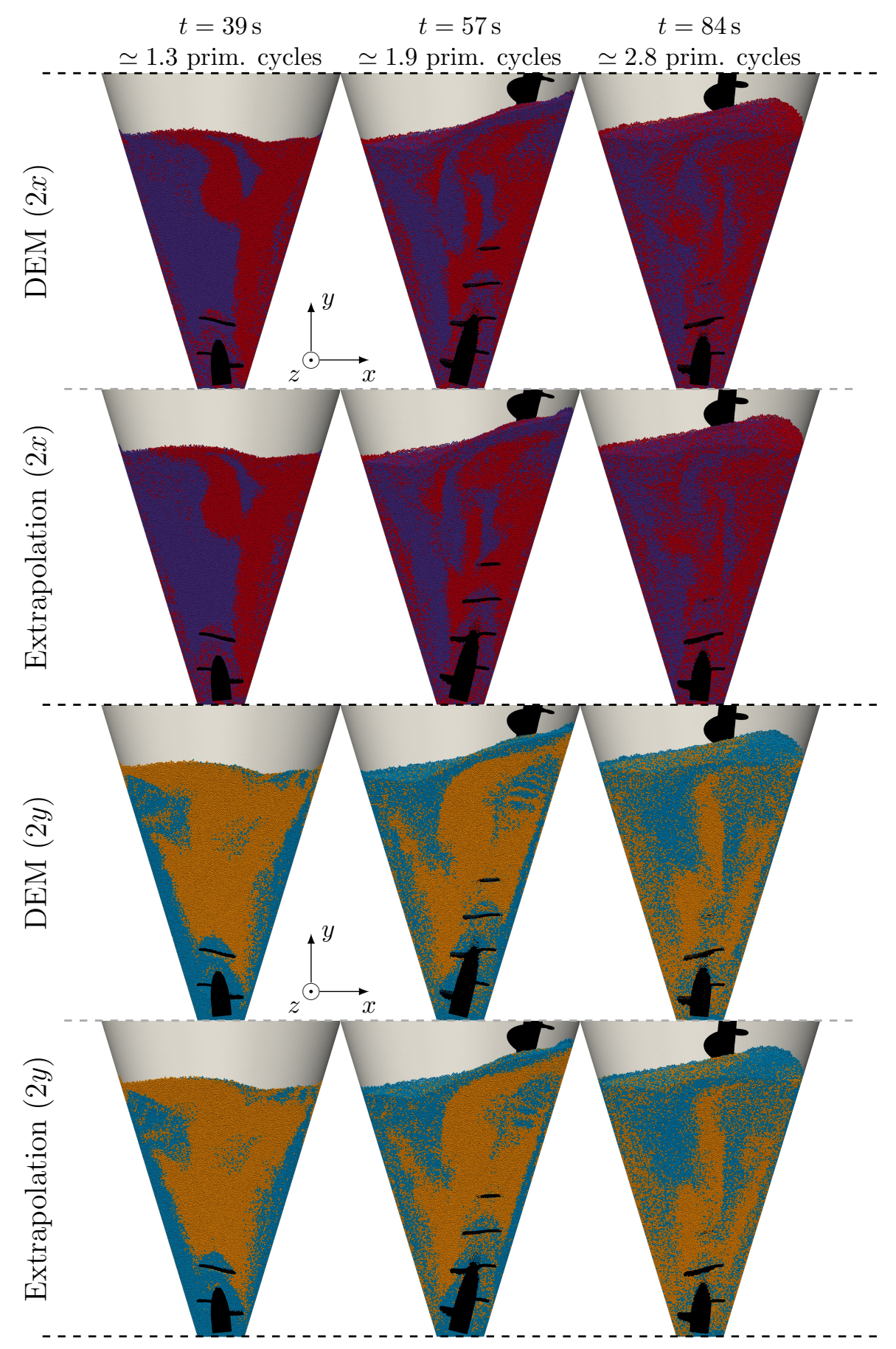

Figure 9: Cross sectional views of the mixer at different stages for the $2 x$ and $2 y$ initial configurations. Comparison between DEM and extrapolated results. 


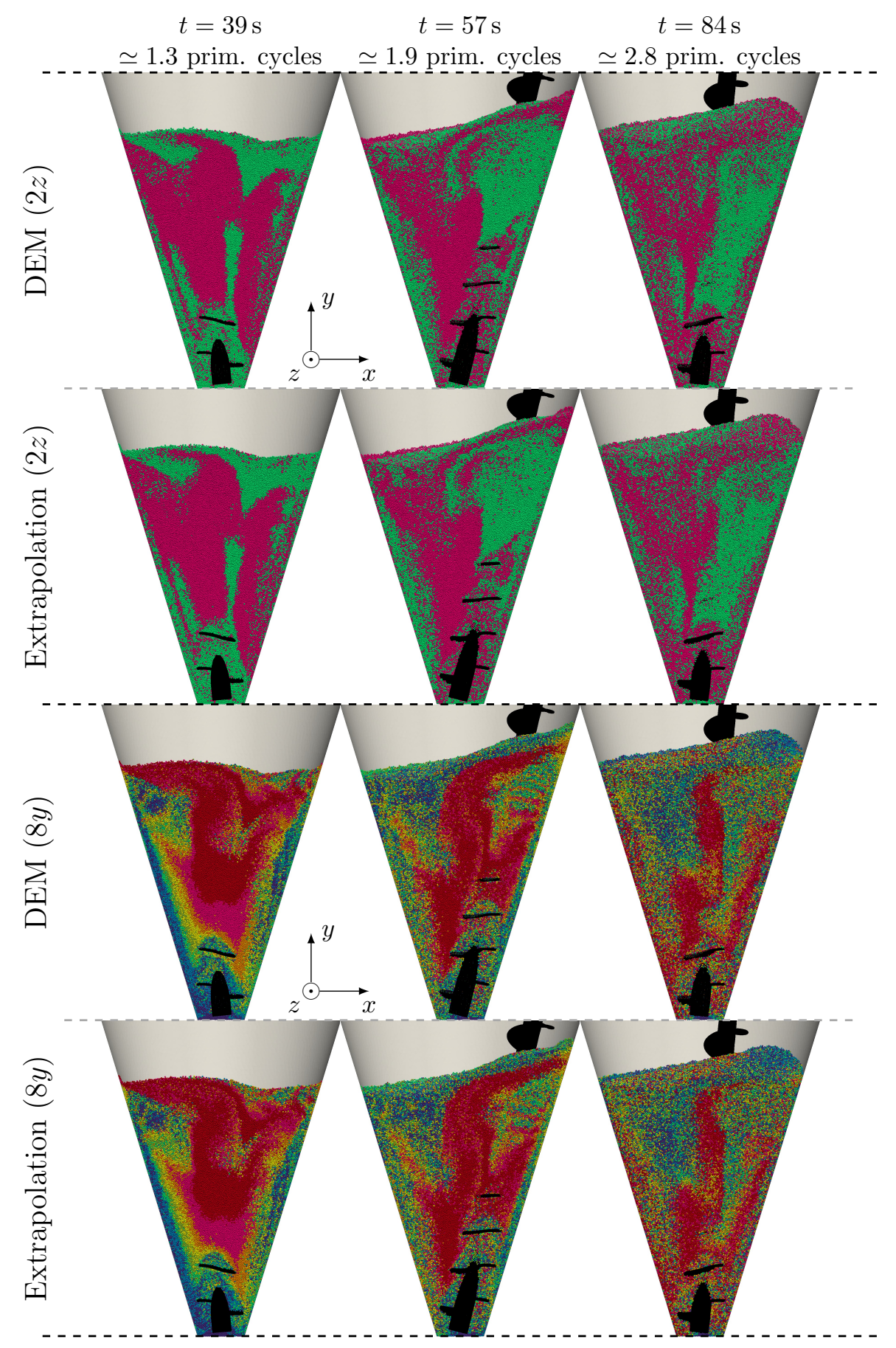

Figure 10: Cross sectional views of the mixer at different stages for the $2 z$ and $8 y$ initial configurations. Comparison between DEM and extrapolated results. 


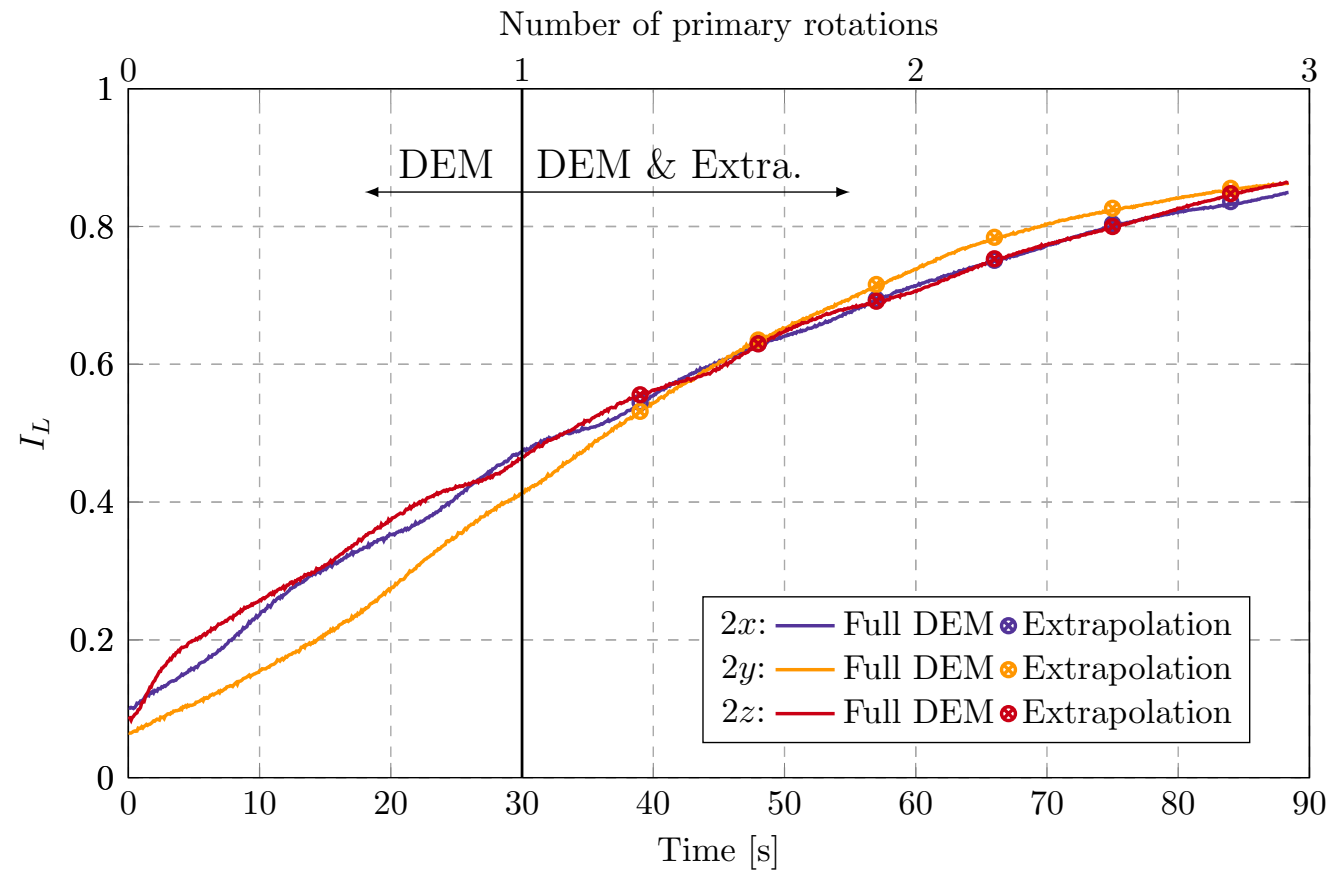

Figure 11: Comparison between extrapolated and DEM Lacey index $I_{L}$. Results for configuration $2 x, 2 y$ and $2 z$.

Table 4: Decile absolute relative dispersion error $\left|\left(d_{\mathrm{disp}}^{\text {extra }}-d_{\mathrm{disp}}^{D E M}\right) / d_{\mathrm{disp}}^{D E M}\right|$

\begin{tabular}{ccccccccc}
\hline D1 & D2 & D3 & D4 & D5 & D6 & D7 & D8 & D9 \\
\hline 0.0876 & 0.179 & 0.278 & 0.394 & 0.549 & 0.814 & 1.31 & 2.27 & 5.11 \\
\hline
\end{tabular}




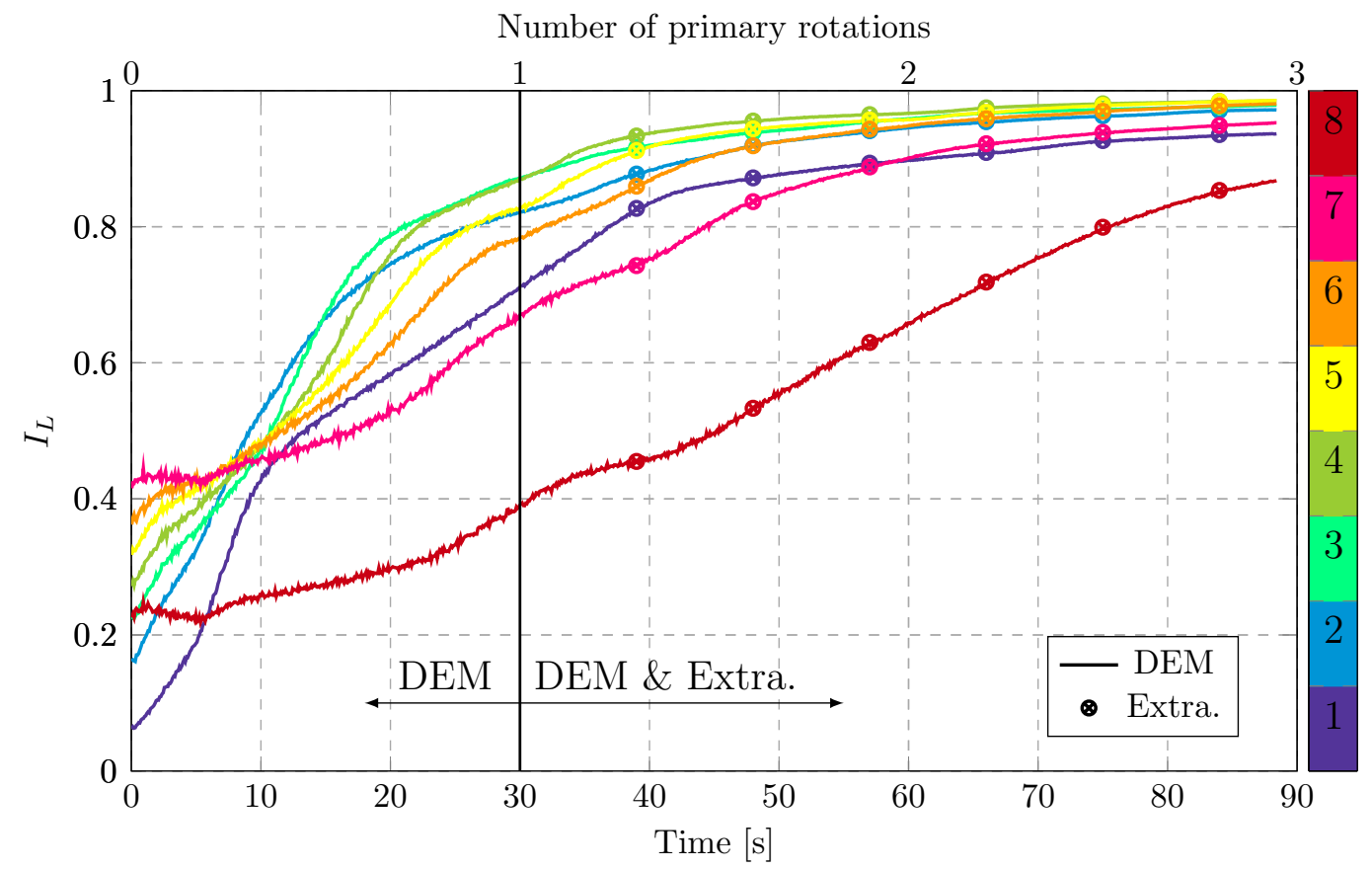

Figure 12: Comparison between extrapolated and DEM Lacey index $I_{L}$. Results for configuration $8 y$. 


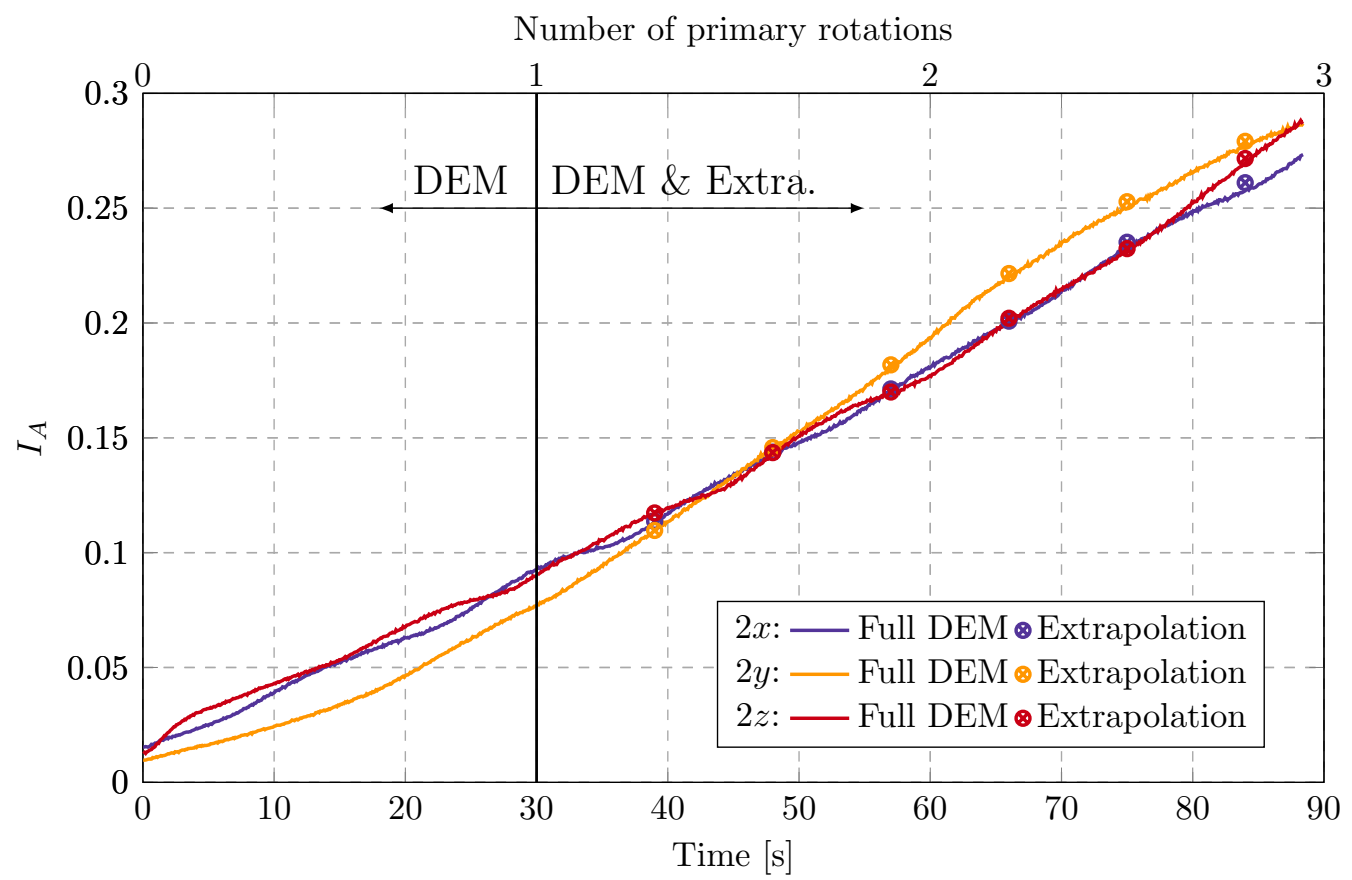

Figure 13: Comparison between extrapolated and DEM Ashton index $I_{A}$. Results for configuration $2 x, 2 y$ and $2 z$. 


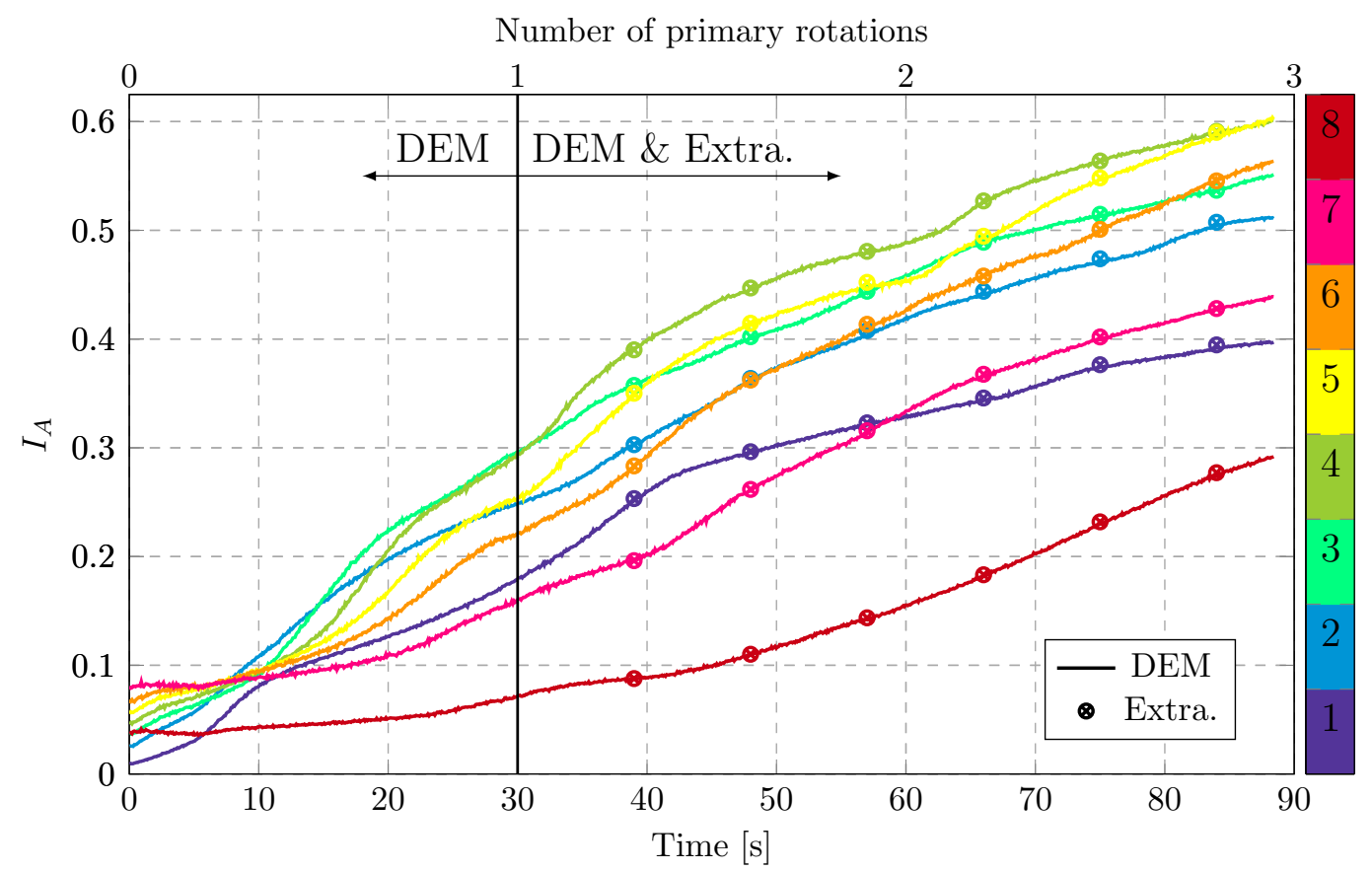

Figure 14: Comparison between extrapolated and DEM Ashton index $I_{A}$. Results for configuration $8 y$.
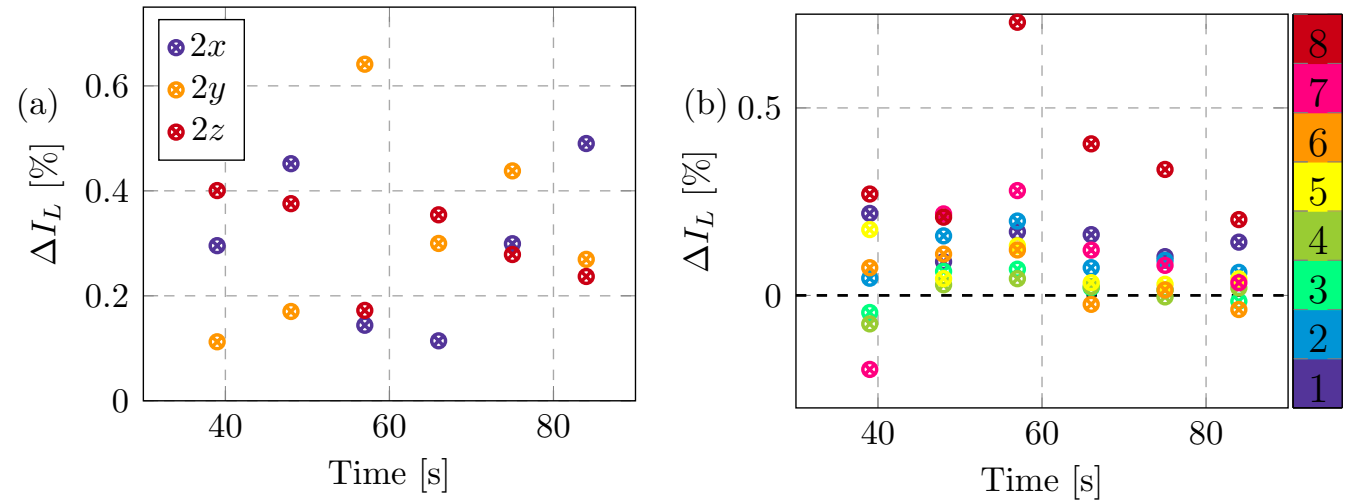

Figure 15: Relative difference for the Lacey index between DEM and extrapolated results for configurations $2 x, 2 y, 2 z$ (left) and $8 y$ (right). $\Delta I_{L}=\left(I_{L}^{\text {extra }}-I_{L}^{\text {DEM }}\right) / I_{L}^{\text {DEM }}$. 

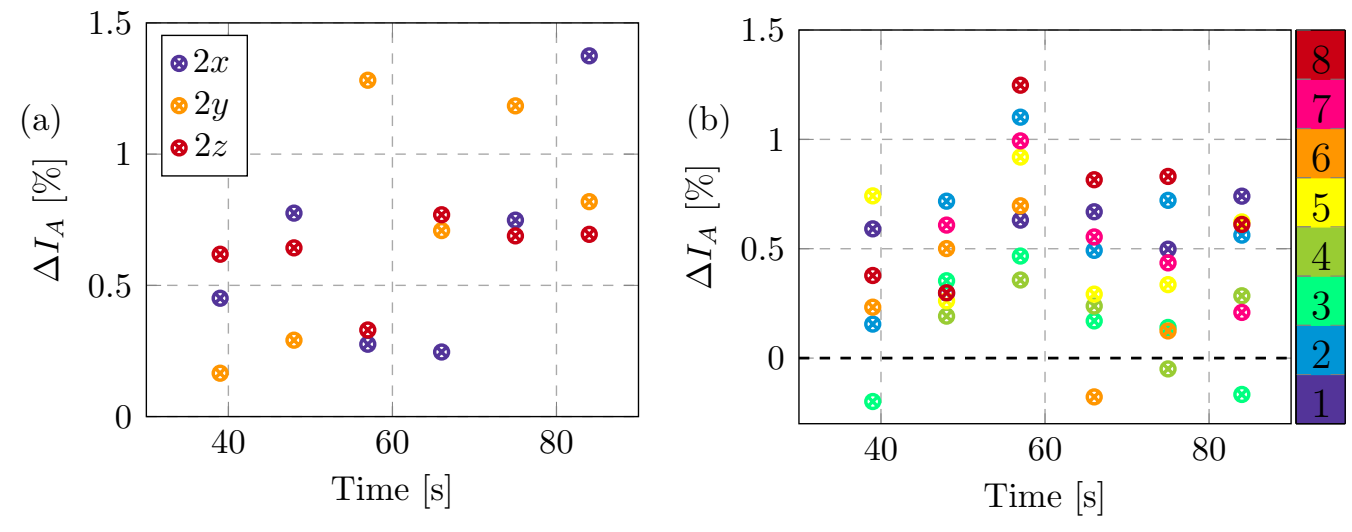

Figure 16: Relative difference for the Ashton index between DEM and extrapolated results for configurations $2 x, 2 y, 2 z$ (left) and $8 y$ (right).. $\Delta I_{A}=\left(I_{A}^{\text {extra }}-I_{A}^{\text {DEM }}\right) / I_{A}^{\text {DEM }}$.

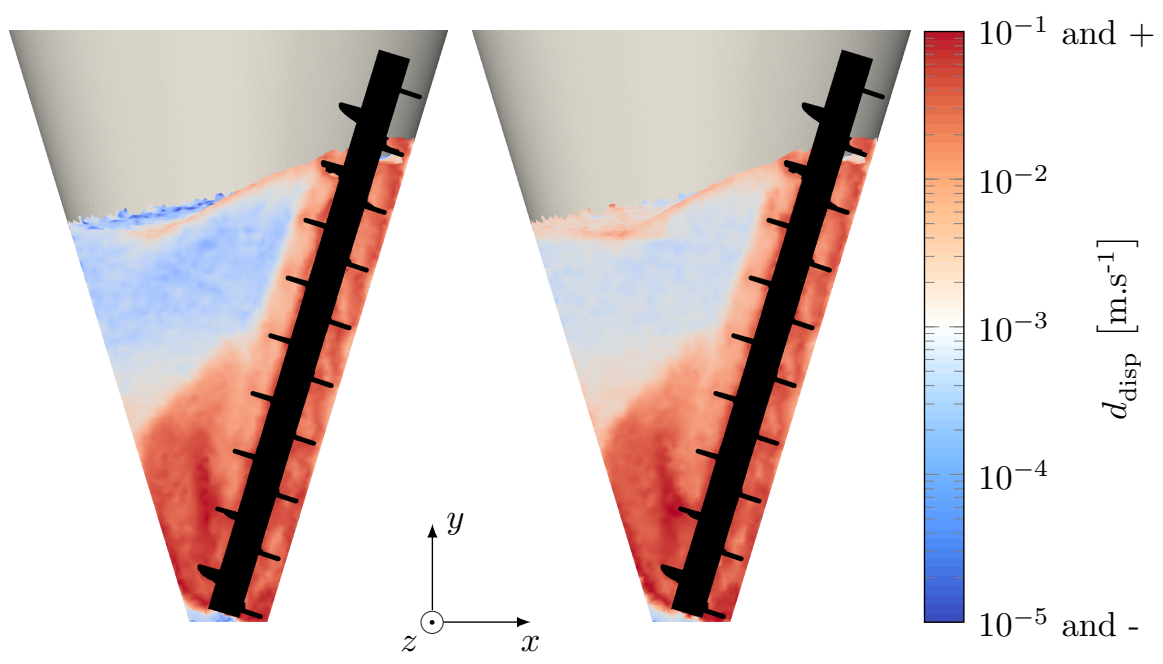

Figure 17: Comparison between dispersion fields for DEM simulation (left) and extrapolation (right) starting from $t_{1}=30 \mathrm{~s}$ with $\Delta t_{\text {disp }}=\alpha T=9 \mathrm{~s}$. 


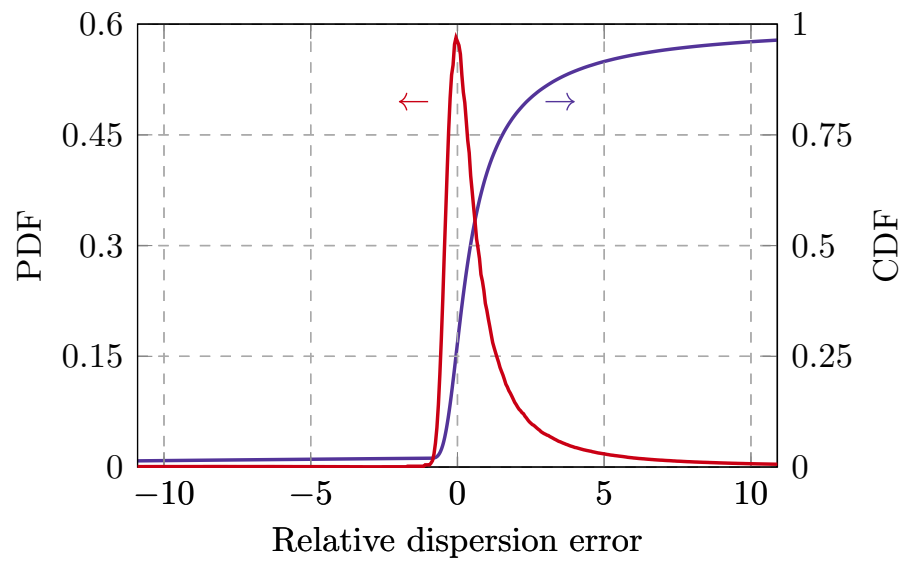

Figure 18: Relative difference of dispersion between DEM and extrapolated results: $\left(d_{\text {disp }}^{\text {extra }}-d_{\text {disp }}^{D E M}\right) / d_{\text {disp }}^{D E M}$ presented as probability density function (PDF) and cumulative distribution function (CDF). Here are plotted $95 \%$ of the values which present the smallest absolute relative dispersion error. Deciles of absolute relative dispersion error are given in Table 4 . 


\title{
Notations
}

\author{
$d_{p}$ Mean diameter of particles $[\mathrm{m}]$ \\ $d_{p}^{\min }, d_{p}^{\max }$ Minimum and maximum diameter of particules $[\mathrm{m}]$ \\ $d_{\text {disp }}$ Dispersion length $[\mathrm{m}]$ \\ $d_{\text {pair }}$ Pairing distance $[\mathrm{m}]$ \\ $i, j$ Indices, identifiers of particles [-] \\ $f$ Pairing function [-] \\ $N$ Number of particles in the mixture [-] \\ $N_{x}, N_{y}, N_{z}$ Number of slice in the $x, y$ and $z$ direction [-] \\ $M_{i}$ Number of particles in the cluster $i$ excluding the central one [-] \\ $p$ Global proportion of the component of interest [-] \\ $\vec{r}_{i}$ Position of the center of particle $i[\mathrm{~m}]$ \\ $R$ Mean radius of particles $[\mathrm{m}]$ \\ $R_{i}$ Radius of particle $i[\mathrm{~m}]$ \\ $t_{0}$ Starting time step for extrapolation [s] \\ $t_{1}$ Ending time step for extrapolation [s] \\ $t_{n+1}$ Extrapolated time step $(n>1)[\mathrm{s}]$ \\ $T$ Time period of the process $[\mathrm{s}]$ \\ $v_{\text {ave }}$ Average translational velocity magnitude of particles $\left[\mathrm{m} . \mathrm{s}^{-1}\right]$ \\ $\alpha$ Multiplier of time period $T$ giving the extrapolation step [-] \\ $\Gamma$ Torque on the screw impeller along its rotation axis [N.m] \\ $\Delta t_{\text {disp }}$ Time interval used to calculate the dispersion [s] \\ $\omega_{a}$ Primary rotation velocity (arm) $\left[\right.$ cycle.s $\left.^{-1}\right]$ \\ $\omega_{s}$ Secondary rotation velocity (screw) $\left[\right.$ cycle. $\left.^{-1}\right]$
}

